\title{
Effects of developmental perfluorooctane sulfonate exposure on spatial learning and memory ability of rats and mechanism associated with synaptic plasticity
}

Yu Wang, Wei Liu, Qian Zhang, Huimin Zhao*, Xie Quan

Key Laboratory of Industrial Ecology and Environmental Engineering (MOE), School of Environmental Science and Technology, Dalian University of Technology, Dalian 116024, China.

Yu Wang, E-mail address: wy-986@163.com

Qian Zhang, E-mail address: zhangqian726@sina.com

Xie Quan, E-mail address: quanxie@ dlut.edu.cn

Wei Liu, E-mail address: liu_wei@dlut.edu.cn

*Huimin Zhao

School of Environmental Science and Technology,

Dalian University of Technology,

Ganjingzi District, Linggong Road 2, Dalian 116024, China.

Tel.: +8641184706263

E-mail: zhaohuim@dlut.edu.cn 


\section{Abstract}

The present study aims to explore the effects of perfluorooctane sulfonate (PFOS) on cognitive function in developing rats and the underlying mechanism associated with synaptic plasticity. Pregnant Wistar rats were fed with 0,5 , and $15 \mathrm{mg} / \mathrm{L}$ of PFOS via drinking water during gestation and lactation. Offspring were exposed to PFOS on prenatal and/or postnatal days by cross-fostering. Spatial learning and memory abilities were tested from postnatal day (PND) 35. We also analyzed the expression pattern of the synaptic plasticity-related genes and proteins in the hippocampus on PND7 and PND35. Results revealed that PFOS exposure reduced the spatial learning and memory abilities of the offspring, particularly of those with prenatal exposure. Meanwhile, protein levels of growth-associated protein-43, neural cell adhesion molecule 1, nerve growth factor, and brain-derived neurotrophic factor decreased on PND35, which are involved in the formation of synaptic plasticity. In contrast, significant increase in gap-43, ncaml, and bdnf genes on the mRNA level was observed on PND7, possibly due to the post-transcriptional mechanism. Results of both behavioral effects and molecular endpoints suggested the high risk of prenatal PFOS exposure. The decline of spatial learning and memory abilities induced by developmental PFOS exposure was closely related to synaptic plasticity.

Keywords: Perfluorooctane sulfonate; Rat offspring; Hippocampus; Prenatal; Postnatal; Synaptic plasticity 


\section{Introduction}

Perfluorooctane sulfonate (PFOS), a lipid- and water-repellent surfactant, is extensively used in both household and industrial applications, such as carpets, leather, paper, and fabric, for more than 50 years (Thompson et al., 2011). Given the bioaccumulation and biomagnification characteristics of PFOS, this surfactant has been detected in various environmental media and is transferred through the food chain to high trophic levels and to humans (Giesy and Kannan, 2001; Lau et al., 2007; Jin et al., 2009). In May 2009, the Stockholm Convention listed PFOS as a persistent organic pollutant.

Toxicological studies in rodents have proven that PFOS can cross the placental and blood-brain barriers (Chang et al., 2009; Borg et al., 2011), thereby causing a potential damage to the central nervous system (CNS) (Austin et al., 2003; Liu et al., 2010a, 2010b) and resulting in developmental delay of neonatal growth (Lau et al., 2003; Fuentes et al., 2007a). However, limited information is available on the behavioral effects of PFOS. No effects on learning and memory abilities were observed in some studies (Lau et al., 2003; Luebker et al., 2005), and slight activity and exploratory behavioral effects occurred when rats and mice were prenatally exposed to PFOS (Fuentes et al., 2007a). PFOS exposure on postnatal day (PND) 10 induced deranged spontaneous behavior in adult mice (Johansson et al., 2008). Regarding the rapid growth of the developing brain, the dose-response relationship of the behavioral effects could be complicated by the corresponding sensitive periods.

Spatial learning and memory are fundamentally dependent on synaptic plasticity, which is the ability of individual synaptic junctions to use or disuse transmission over synaptic pathways. Our previous study, employing a genome-wide DNA analysis, showed that the differentially expressed genes induced by PFOS in rat cerebral cortex are involved in synaptic plasticity (Wang et al., 2010). Moreover, several microRNAs critical in synaptic transmission were also significantly altered by PFOS (Wang et 
al., 2012). Therefore, further research is required to evaluate the effects of PFOS on synaptic plasticity and its role in the PFOS-induced interference of cognitive ability.

During the neurodevelopmental period, several critical proteins are involved in synaptic plasticity. Both GAP-43 and NCAM1 are necessary membrane proteins for the neuronal development (Fields and Itoh, 1996; Oestreicher et al., 1997), while nerve growth factor (NGF) and brain derived neurotrophic factor (BDNF) are important members of the neurotrophin family, which regulate neurons survival, differentiation, growth, and hippocampal LTP (Fischer et al., 1991; Patterson et al., 1992). These synaptic plasticity related proteins play important roles in regulation and repair of the nervous system, whose alteration could be another or contributing mechanism behind the observed cognitive ability impairments after exposure to PFOS. But few studies reported that they were associated with the developmental neurotoxic effects of PFOS. Single neonatal exposure (PND10) or exposure from gestational day 1 (GD1) to PND14 caused up regulation of GAP-43, and NCAM1, respectively (Johansson et al., 2009; Wang et al., 2011). The evaluation of key molecular factors in synaptic plasticity would favor a better understand of the potential neurotoxicological mechanism of PFOS.

We aimed to identify the effects of PFOS exposure during prenatal and postnatal periods on cognitive ability, as well as to elucidate the mechanism underlying PFOS-induced neurodevelopmental damage associated with synaptic plasticity. Critical proteins and genes (GAP-43, NCAM1, NGF, and BDNF in the hippocampus) involved in synaptic plasticity were evaluated in hippocampus, an important functional area related to learning and memory abilities.

\section{Materials and methods}

\subsection{Ethical approval of the study protocol}


The study protocol was approved by the School of Environmental Science and Technology, Dalian University of Technology (Dalian, China).

\subsection{Reagents}

PFOS (CAS number 2795-39-3, purity $\geq 98 \%$, Sigma-Aldrich, USA) solution was prepared using 2\% Tween 20 (purity $\geq 97 \%$, Amresco, USA) in deionized water at concentrations of 2.5 and $7.5 \mathrm{~g} / \mathrm{L}$. The stock PFOS solution was diluted 500-fold by sterile tap water and used as the drinking water in the 5 and $15 \mathrm{mg} / \mathrm{L}$ exposure groups. The control group was fed drinking water containing $0.004 \%$ Tween 20.

\subsection{Animal treatment and sample collection}

Wistar rats weighing $180 \mathrm{~g}$ to $220 \mathrm{~g}$ were fed with standardized rodents feed particles and tap water under a 12:12 hours of light: dark pattern at a temperature of $24 \pm 2{ }^{\circ} \mathrm{C}$ and a relative humidity of 50-60\%. Pregnant rats were randomly divided into control group (CC), low and high dose treatment group, fed with drinking water containing 0, 5, $15 \mathrm{mg} / \mathrm{L}$ PFOS, respectively. Details are presented in the Supplementary Information (SI). The PFOS concentrations in the serum of dams and in the hippocampus of rat offspring were analyzed to further confirm the internal exposure dose (SI, Table S1, S2). On PND1, litter parameters were recorded (SI, Table S3), pups in the control and exposed groups were cross-fostered to further establish the following groups: $\mathrm{CC}$ without exposure; continuous exposure to 5 and $15 \mathrm{mg} / \mathrm{L}$ of PFOS (TT5, TT15); only postnatal exposure at 5 and $15 \mathrm{mg} / \mathrm{L}$ of PFOS (CT5, CT15); and only prenatal exposure at 5 and $15 \mathrm{mg} / \mathrm{L} \mathrm{PFOS} \mathrm{(TC5,} \mathrm{TC15).}$ 
Pups were sacrificed on PND7 and PND35. In each rat, the hippocampus was dissected from the brain and immediately frozen at $-80^{\circ} \mathrm{C}$ before gene and protein analyses. Spatial learning and memory abilities were evaluated by Morris water maze (MWM) starting from PND35.

\subsection{Morris Water Maze (MWM)}

MWM was used to evaluate the spatial learning and memory abilities of pups (Morris et al., 1982). The MWM tests comprised 1 day (d) of visible platform tests, $7 \mathrm{~d}$ of hidden platform tests, and a probe trial $24 \mathrm{~h}$ after the last hidden platform test. Details are shown in SI.

\subsection{Quantification of protein levels of GAP-43, NCAM1, NGF, and BDNF}

Each hippocampal sample from individual rat was weighed and homogenized in ice-cold lysis buffer containing $137 \mathrm{mM} \mathrm{NaCl}, 20 \mathrm{mM}$ Tris- $\mathrm{HCl}$ (pH 8.0), $1 \mathrm{mM}$ phenylmethylsulfonyl fluoride, $10 \mathrm{mg} / \mathrm{ml}$ of aprotinin, $1 \mathrm{mg} / \mathrm{ml}$ of leupeptin, $0.5 \mathrm{mM}$ sodium vanadate, $10 \%$ glycerol, and $1 \%$ nonyl phenoxypolyethoxylethanol. Homogenized samples were then centrifuged at 14,000 $g$ for $15 \mathrm{~min}$ at $4{ }^{\circ} \mathrm{C}$. Supernatant was removed and assayed immediately. The total protein amounts were determined using the total protein assay kit (Nanjing Jiancheng Bioengineering Institute, China). The GAP-43, NCAM1, NGF, and BDNF protein levels were measured by rat ELISA kit (Nanjing Jiancheng Bioengineering Institute, China) according to the manufacturer's instructions. Concentrations of all the samples were higher than the detection limit of these four proteins.

\subsection{Real-time quantitative RT-PCR analyses}


RNA was isolated from the hippocampus of individual rats. The RNA was then reverse-transcribed to cDNA using TransScript All-in-One First-Strand cDNA Synthesis SuperMix for qPCR kit (Transgene, China). Rat-specific primers were designed for the gap-43, ncaml, ngf, and bdnf genes. The sequences of the primers were designed using Primer Premier 5.0 software (Premier Biosoft International, USA). The housekeeping gene $\beta$-actin was assayed as an internal control as described by Liu et al. (2010b) (SI, Table S4). $\beta$-actin was chosen as a control because it was widely used and did not vary significantly between the brain areas and ages in the developing brain of rat compared with other housekeeping genes (Polesskaya et al., 2007). The PCR amplification protocol was $30 \mathrm{~s}$ at $94{ }^{\circ} \mathrm{C}$, followed by 40 cycles at $94{ }^{\circ} \mathrm{C}$ for $5 \mathrm{~s}$, and $60{ }^{\circ} \mathrm{C}$ for $30 \mathrm{~s}$. Each sample analysis was performed in triplicate. The expression level of the target gene was normalized to that of $\beta$-actin. The fold change of the target genes was analyzed by $2^{-\Delta C_{T}}$ method (Livak and Schmittgen, 2001).

\subsection{Statistical analyses}

Data were analyzed using SPSS 16.0 software (SPSS, Chicago, IL, USA) and expressed as mean \pm standard error. One-way analysis of variance (ANOVA) followed by least significant difference (LSD) was used to determine the differences among groups of the gene and protein expression levels. For the hidden platform test, the latencies and distances were averaged and analyzed using repeated measures and multivariate ANOVA followed by LSD post-hoc test of the general linear mode. For the visible platform test and probe trials, the values were averaged and analyzed using one-way ANOVA followed by Duncan's multiple-range test. Statistical significance level was set at $p<0.05$.

\section{Results and discussion}




\subsection{Effects of PFOS on spatial learning and memory abilities of pups}

In the visible platform tests, no significant statistical difference was observed in the swimming speeds and the time to reach the visible platform (SI, Fig. S1). This finding indicated that PFOS exposure did not affect the visual and motor functions of the rat offspring.

The learning ability of the offspring was reflected by the hidden platform test in MWM. The results showed that the escape latency of the rat pups from the exposure groups was longer than that of the CC group, where the highest increase was observed in the prenatal high-dose group, TC15. Besides, significant difference was observed between TC15 and CC groups from PND36 to PND41. The escape latency also significantly increased in the prenatal low-dose group, TC5, on PND37 and PND38. For the continuous exposure group, decreased escape latency was observed in the high-dose group, TT15, on PND37 and PND38. No significant change in the escape latency occurred in the continuous low-dose group, TT5. For the postnatal exposure group, moderate increase in escape latency appeared in the low and high-dose groups, CT5 and CT15 (Table 1). Similarly trends appeared in the results of the escape distances (Fig. 1). The memory ability of the offspring in the original platform was reflected by the probe trial test. The results showed that the time spent in the target quadrant by pups in the exposure groups was shorter than that spent by the CC group, with a significant difference in the TT15 group (Fig. 2A). Meanwhile, the numbers of platform crossings in the TT15 group were also significantly smaller than that in the CC group (Fig. 2B).

\section{Table 1}

Escape latency of rat offspring after PFOS exposure analyzed by MWM. 


\begin{tabular}{|c|c|c|c|c|c|c|c|c|}
\hline & & PND35 & PND36 & PND37 & PND38 & PND39 & PND40 & PND41 \\
\hline $\mathrm{CC}$ & 8 & $77.27 \pm 10.82$ & $41.48 \pm 4.60$ & $23.76 \pm 3.04$ & $17.76 \pm 2.04$ & $23.64 \pm 3.57$ & $16.59 \pm 1.94$ & $17.60 \pm 1.43$ \\
\hline TT5 & 6 & $80.10 \pm 4.45$ & $49.21 \pm 4.45$ & $19.72 \pm 3.20$ & $22.49 \pm 5.51$ & $21.96 \pm 5.57$ & $15.14 \pm 1.59$ & $15.44 \pm 2.92$ \\
\hline TT15 & 10 & $85.88 \pm 11.60$ & $58.49 \pm 12.73$ & $44.13 \pm 4.75 * *$ & $29.75 \pm 5.23 *$ & $26.19 \pm 3.78$ & $22.74 \pm 5.31$ & $23.78 \pm 2.95$ \\
\hline TC5 & 10 & $80.02 \pm 13.76$ & $51.38 \pm 7.59$ & $35.4 \pm 23.81$ & $38.82 \pm 5.07 *$ & $27.24 \pm 3.76^{*}$ & $20.41 \pm 2.19$ & $23.65 \pm 1.65$ \\
\hline TC15 & 10 & $91.47 \pm 8.54$ & $65.66 \pm 16.56^{*}$ & $49.41 \pm 7.12 * *$ & $35.69 \pm 3.18 *$ & $41.50 \pm 1.82 * *$ & $29.61 \pm 4.08 * *$ & $31.01 \pm 1.31 *$ \\
\hline CT5 & 9 & $83.92 \pm 8.68$ & $48.45 \pm 6.22$ & $39.99 \pm 6.22 *$ & $28.14 \pm 2.60 *$ & $24.17 \pm 1.87$ & $25.36 \pm 2.73$ & $22.67 \pm 3.10$ \\
\hline CT15 & 10 & $80.08 \pm 9.26$ & $57.80 \pm 8.17$ & $35.57 \pm 3.77$ & $28.63 \pm 3.66^{*}$ & $24.15 \pm 3.94$ & $20.53 \pm 2.51$ & $21.29 \pm 2.38$ \\
\hline
\end{tabular}

Data is expressed as mean \pm SE. * Statistically significant $(p<0.05)$ difference from CC group.

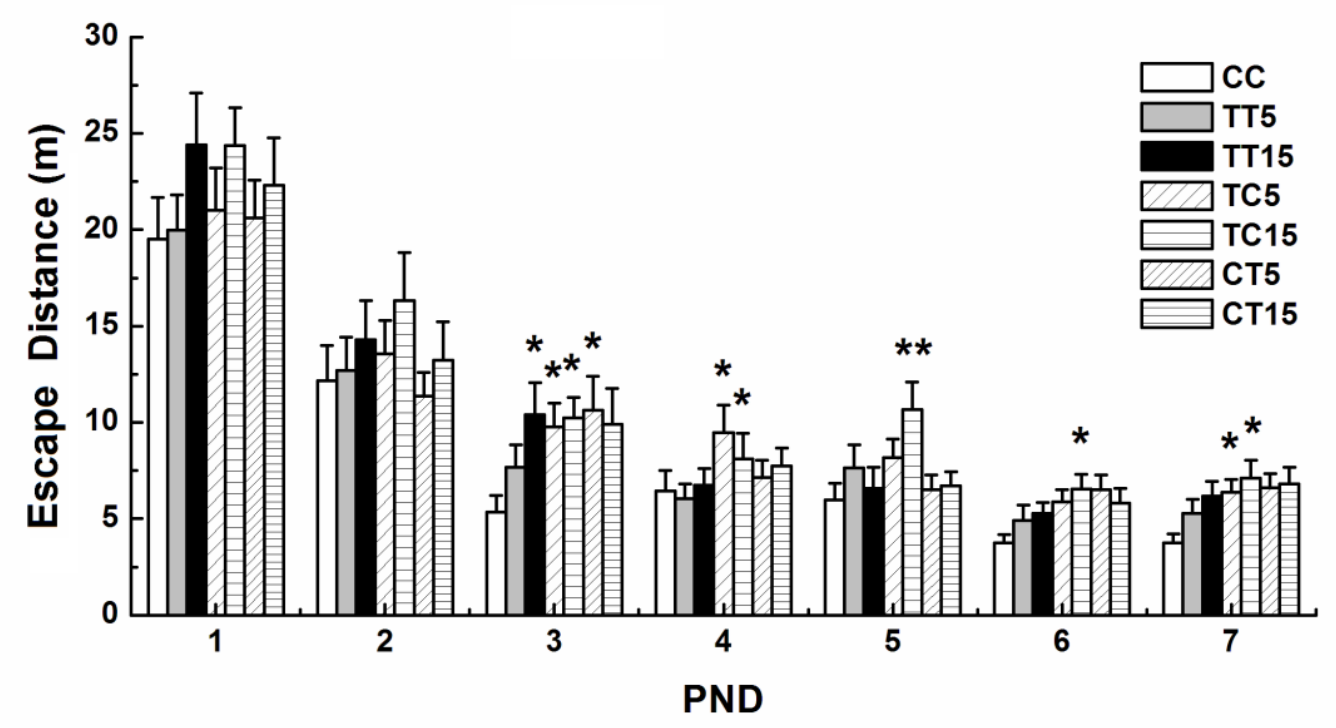

Fig. 1. Swimming distances to find the hidden platform over 7 consecutive training days. Data is expressed as mean \pm SE. * Statistically significant $(p<0.05), * *$ Statistically significant $(p<0.01)$ difference from CC group at the same time point. 

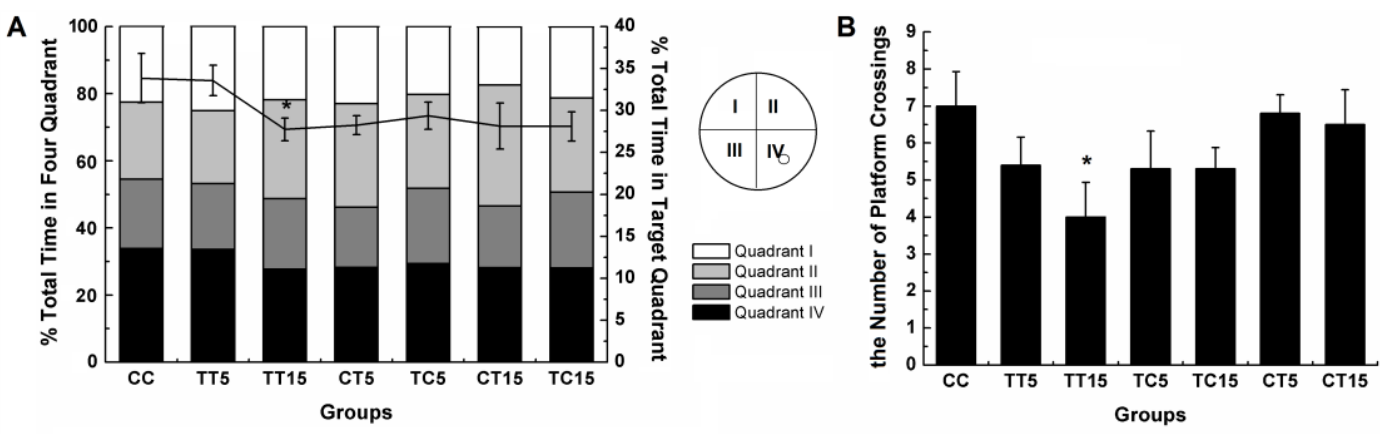

Fig. 2. Percentage of swimming time in different directions at 8th training day. A, Histograms showing the percentage of time spent in four quadrants and target quadrants in various groups, and the linear chart showing the percentage of time spent in the target quadrant in different groups. Data is expressed as mean \pm SE. Quadrants IV represents the target quadrant. B, the number of platform crossings in different groups. * Statistically significant $(p<0.05)$ difference from CC group.

Although the hippocampus PFOS concentrations in the TC15 group were much lower than those in the continuous exposure and postnatal high-dose groups (SI, TableS3), the mean escape distances were significantly longer in the TC15 group than in the CC group from PND36 to PND41. These findings suggested that strong neurotoxic effects occurred in the developing nervous system with prenatal PFOS exposure. The time spent in the TT15 groups exhibited significant change compared with that in the CC group during the probe test. The results indicated that the spatial learning and memory abilities were impaired by PFOS exposure, and prenatal PFOS exposure resulted in much serious decline of learning abilities of the offspring.

Given that both negative and positive behavioral effects were reported, PFOS exposure during different life periods resulted in various patterns of alteration in mammalian behavior. In a study by Fuentes et al. (2007b), three-month-old mice were administered $3 \mathrm{mg}$ PFOS/kg/d via gavage for four consecutive weeks, and a slight deficit in spatial memory was observed. Johansson et al. (2008) 
reported that neonatal exposure to 1.4 or $21 \mathrm{mmol} / \mathrm{kg}$ body weight of PFOS on PND10 caused changes in spontaneous behavior and habituation in mice. By contrast, offspring exposed to $1.0 \mathrm{mg} / \mathrm{kg} / \mathrm{d}$ PFOS from GD0 to PND20 did not differ significantly from the control group in learning and memory abilities (Butenhoff et al., 2009). The complex physiological activities during the developmental processes might lead to different results, such as establishment of blood-brain barrier and a period of rapid brain growth called "brain growth spurt" (BGS) (Semple et al., 2013). Neonatal PFOS exposure may pose risk to the CNS of the rats, whereas "exposure time window" is an important factor. Therefore, it is of significance to evaluate the effects of the exposure period on the PFOS-induced neurotoxicity employing the cross-foster model. In addition, the expression patterns of key proteins and genes were analyzed on two critical time points, PND7 and PND35, to reveal the molecular mechanisms underlying the effects of PFOS during brain development. PND7 represented one sequential point during the maturation of the blood-brain barrier, and PND35 is the time for brain maturation (Wang et al., 2010).

\subsection{Effects of PFOS on protein levels of GAP-43, NCAM1, NGF, and BDNF}

The targeted protein levels in the hippocampus are shown in Fig. 3. GAP-43 in the hippocampus was not significantly different among the exposure groups on PND7. On PND35, GAP-43 showed significant increase in the TT15 group, whereas GAP-43 levels were significantly lower in the TC and CT groups than in the CC group. The decreased memory ability in the TT15 group was accompanied by the significant elevation of GAP-43 on PND35. GAP-43 plays a positive role in mediating neurite outgrowth and regeneration and can be increased by 20 to 100 folds after peripheral nerve injury as a result of CNS system self-protection (Zhang et al., 2012). Other studies have also found associations 
between PFOS exposure and changes in GAP-43 amount. The GAP-43 protein level significantly increased in the hippocampus of neonatal mice administered with a single oral dose of PFOS on PND10 (Johansson et al., 2009). Similar result appeared when neonatal rats were exposed to PFOS from GD1 to PND14 (Wang et al., 2011). It has been also reported that depletion of GAP-43 markedly prevents neurite and growth cones formation (Aigner and Caroni, 1995). Combined with the present results in the continuous exposure groups and prenatal/postnatal exposure groups, PFOS exposure could stimulate GAP-43 expression, or might also pose a negative effect on this protein expression, which may be dependent on both the PFOS dose and the exposure time. However, the decline in memory ability in TT15 groups suggested that the increase in GAP-43 levels failed to result in an effective positive response. The result indicated that PFOS-induced behavioral defects might be associated with the alteration of GAP-43 levels.
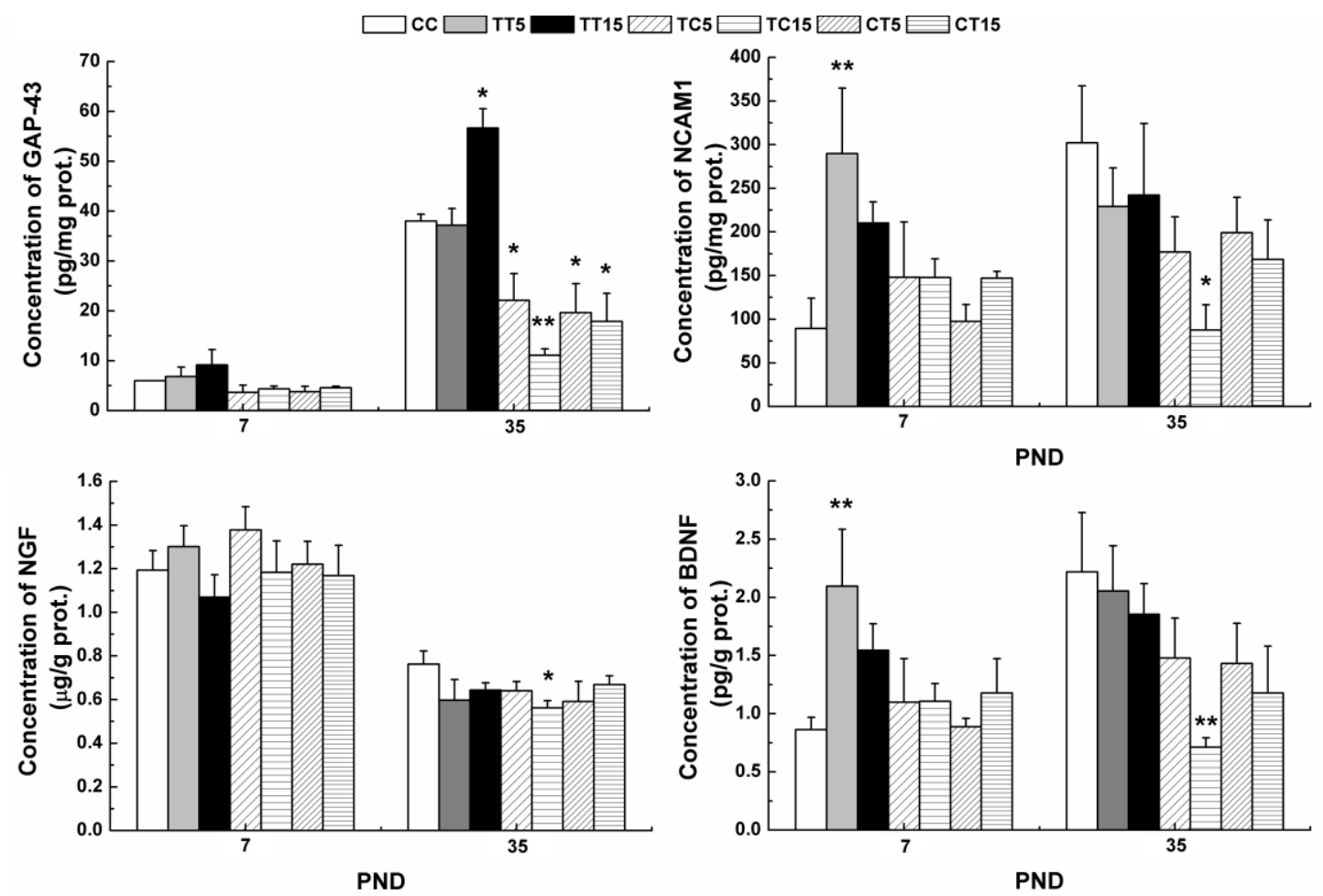
Fig. 3. Effects of PFOS on levels of GAP43, NCAM1, NGF, and BDNF proteins in hippocampus on PND7 and PND35. $\mathrm{n}=3$. * Statistically significant $(p<0.05)$, ** Statistically significant $(p<0.01)$ difference from CC group.

As another necessary membrane protein for neuronal development, NCAM1 was expressed at a significantly higher level in the TT5 group than in the CC group on PND7 with no significant differences in other treatment groups. However, the NCAM1 amount exhibited a decreasing trend and was significantly reduced in the TC15 group on PND35. NCAM1 are likely candidate molecules that participate in synaptogenesis in neuronal plasticity. Low concentrations of NCAM1 lead to defects in synaptic stabilization and deficits in learning ability (Dityater and Schachner, 2003; Petridis et al., 2004; Bukalo et al., 2004). Thus, PFOS inhibited the NCAM1 expression and could damage synaptic plasticity, which could lead to negative effects on spatial learning and memory.

No significant difference was found for NGF amount between the PFOS treatment and CC groups on PND7. On PND35, NGF showed a slightly downward trend and was significantly reduced in the TC15 group. Changes in BDNF amount exhibited a similar pattern with NGF. BDNF significantly increased in the TT5 group on PND7 with no significant differences in other groups. BDNF showed a downward trend on PND35 and was reduced significantly in the TC15 group. As important neurotrophins, NGF and BDNF are critical for the survival and maintenance of sympathetic and sensory neurons. In vivo experiments showed that nerve-injured rats administered with NGF at $80 \mathrm{ng} / \mathrm{d}$ for three weeks exhibited significant sensorimotor recovery (Kemp et al., 2011). In vitro experiments confirmed that NGF could protect retinal pigment epithelial cells against $\mathrm{H}_{2} \mathrm{O}_{2}$-induced apoptosis (Cao et al., 2011). BNDF is usually upregulated in response to injury in the adult brain (Hughes et al., 1999). The BDNF 
protein level significantly decreased on PND7 and PND14 when neonatal rats were exposed to PFOS from gestational day 1 (GD1) to PND14 (Wang et al., 2011). BDNF knockout mice showed a strong impairment in LTP, suggesting that decreased hippocampal BDNF may be directly related to cognitive deficits and spatial learning (Korte et al., 1995; Heldt et al., 2007). The altered levels of NGF and BDNF could be one of the mechanisms of behavioral defects induced by PFOS.

These four synaptic plasticity-related proteins were significantly decreased in the TC15 group on PND35. These findings were attributed to the decline of the spatial learning ability. Thus, multiple proteins associated with synaptic plasticity are subject to change after PFOS exposure, which would consequently result in damages during differentiation and maturation of neurons. Such damages would finally be manifested as decreased learning and memory abilities. However, the changes in the levels of these proteins on PND7 were different from those on PND35, thereby reflecting a time-dependent neurotoxicity of PFOS during brain development. In rat offspring, BGS existed in the developing brain within a span of the first 3 to 4 weeks of life and peaking at around PND10 (Johansson et al., 2009). This period is associated with numerous biochemical changes, and the relatively high cell activity may be one reason why protein did not change significantly on PND7. Upregulation of NCAM1 and BDNF proteins in the TT5 group on PND7 played a self-defensive role against PFOS neurotoxicity, but such proteins could not be maintained at a high level. The increase in the levels of these proteins failed to restore the learning and memory abilities. All four proteins significantly decreased in the TC15 group on PND35. Despite the absence of PFOS exposure during postnatal period, similar injuries on the proteins were observed in the prenatal exposure groups compared with the postnatal exposure groups, thereby highlighting the health risk of prenatal PFOS exposure. These results indicated the serious risk of prenatal PFOS exposure, and further studies on embryonic exposure to PFOS should be performed. 


\section{3. gap-43, ncaml, ngf, and bdnf transcripts}

The expression of the four genes ( $g a p-43, n c a m 1, n g f$, and $b d n f)$ in the hippocampus was examined. Gap-43 was significantly upregulated in the TT5 and CT15 groups on PND7 (Fig. 4). The gap-43 gene was significantly higher in the TC5 group than in the CT5 group. These results further verified the risk of prenatal PFOS exposure at transcriptional level. The enhancement of gap-43 gene expression indicated a protective mechanism against PFOS neurotoxicity. However, with the continuous exposure to PFOS and the growth of pups, no significant change appeared on PND35. PFOS might induce self-protection by increasing gap-43 expression at the transcriptional level, but failing to enhance the protein amount.

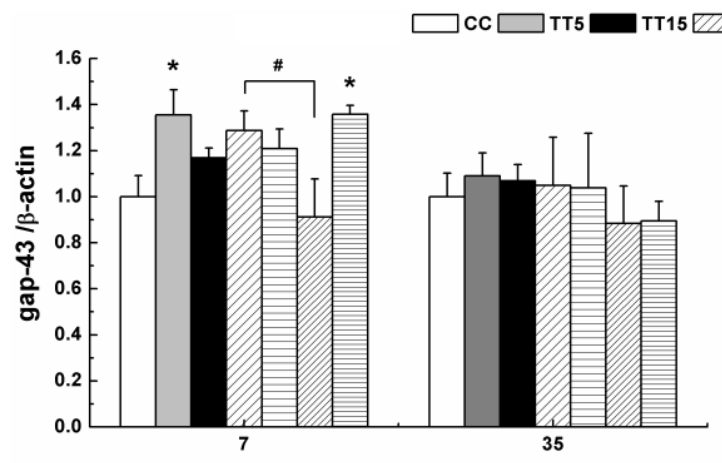

PND
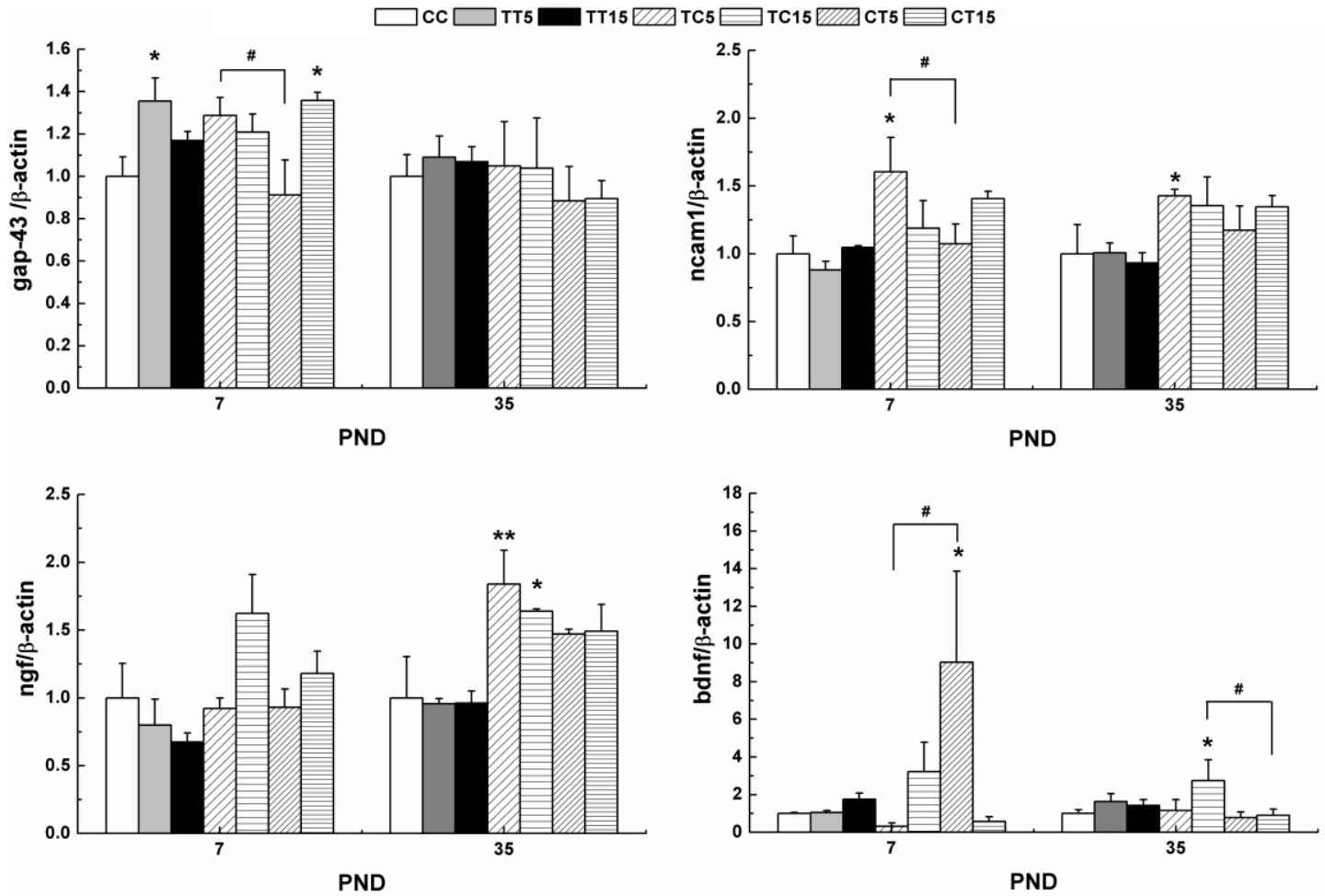
Fig. 4. Effects of PFOS on expression of gap-43, ncaml, $n g f$, and bdnf genes in hippocampus on PNDs 7 and 35. $\mathrm{n}=3$. * Statistically significant $(p<0.05)$, ** Statistically significant $(p<0.01)$ difference from CC group. ${ }^{\#}$ Statistically significant $(p<0.05)$ difference between TC group and CT group.

On PND7, ncam1 level in the TC5 group was significantly higher than that in the CT5 group. Additionally, ncaml levels were upregulated on PND35 with significant changes in the TC5 group (Fig. 4). Upregulation of ncaml expression on PND35 would improve memory and defense against neurotoxicity. However, the corresponding protein amount failed to increase, thereby resulting in impaired synaptic plasticity.

No significant change in $n g f$ expression was observed in cross-fostered groups on PND7. This gene was significantly upregulated in TC5 and TC15 groups on PND35. This finding indicated that prenatal exposure poses more serious risk to the nervous system than postnatal exposure. Bdnf expression was upregulated on PND7, and its expression remarkably increased by 9.2 folds in the CT5 group. On PND35, bdnf expression was upregulated and significantly increased in the TC15 group compared with CC and CT15 groups (Fig. 4). In the cross-fostered groups, bdnf genes also showed non-monotonic dose-response relationship with PFOS concentrations. This finding confirmed the self-protection response at the transcriptional level with no positive effect against PFOS-induced injury at the protein level.

There have been conflicting results on mRNA and protein expressions because of many cellular and physical processes. Integrative analysis of these two data types could provide additional insights into the metabolic mechanisms underlying complex biological systems (Nie et al., 2006). PFOS-induced changes on the mRNA level were different from those on the protein level, suggesting the importance 
of post-transcriptional or translational mechanisms in response to PFOS exposure. Transcription and translation are far from having a linear and simple relationship (Maier et al., 2009). RNA secondary structure and ribosomal density may change translation efficiency, while regulatory proteins and sRNAs can act as translational modulators. Besides, protein stabilization, and mRNA distribution might also be involved on the regulation level. Previous reports find only a weak correlation between the respective abundances of mRNA and proteins. (Gygi et al., 1999; Nie et al., 2006; Medina et al., 2013). In the present study, the targeted gene levels increased, but the corresponding proteins did not change significantly or slightly decreased on PND7. The targeted gene levels were also upregulated on PND35, whereas the protein levels exhibited a downward trend with significant differences in the TC15 group. The difference in the temporal pattern of the gene and protein expressions suggested that changes in mRNA levels were more sensitive than those in proteins, which occurred earlier. However, the increase in gene levels failed to enhance the protein amount. PFOS might also influence many factors of post-transcription and translation, which eventually led to the inhibition of proteins.

The decreased learning and memory abilities are related to some neurodegenerative diseases, such as Alzheimer's and Parkinson's diseases. PFOS exposure may cause early occurrence of neurodegenerative diseases, which is not limited to the elderly, but no epidemiological results are currently available. The occurrence of neurodegenerative diseases has significantly increased recently (Nussbaum and Ellis 2003), and their association with environmental pollution from heavy metals and organic pollutants has garnered widespread attention (Fischer et al., 2008; Andersen et al., 2009). The effects of PFOS on the impairment of cognitive ability, further confirmed by the changes on the molecular level, suggesting that the evaluation of the association between PFOS exposure and the health risk of neurodegenerative diseases, is necessary. 
The current study is the first to use the cross-foster model to evaluate the effects of PFOS on spatial learning and memory abilities in rat offspring. The results showed that prenatal and postnatal PFOS exposure resulted in the deficit in spatial learning and memory abilities of the offspring. Meanwhile, the amount of several key proteins associated with synaptic plasticity were also be repressed by PFOS exposure. Some changes occurred in the corresponding gene levels. The gene levels tended to increase during the early development period, but such increase was not maintained when the level of PFOS exposure increased. The gene enhancement also failed to positively regulate the protein amount, suggesting a post-transcriptional or transcriptional effect. Furthermore, results of both behavioral effects and molecular endpoints suggested that the prenatal period is the critical stage of the PFOS-induced neurotoxicity, thereby indicating the risk of exposure during the neurodevelopmental processes of fetuses and neonates. Exposure to PFOS during the development period caused the expression alteration of synaptic plasticity-related genes and proteins in the hippocampus of the offspring, which could lead to a decline in spatial learning and memory abilities. 
The paper is to commemorate late Prof. Dr. Yihe Jin (1959-2013), who has devoted his whole life to the scientific research, and contributed greatly to the present research.

\section{Acknowledgments}

The study is financially supported by National Natural Science Foundation of China (21177020, 81102096, NSFC-JST 21261140334) and the Fundamental Research Funds for the Central Universities (DUT12LK13). 


\section{References}

Aigner, L., Caroni, P., 1995. Absence of persistent spreading, branching, and adhesion in GAP-43-depleted growth cones. J. Cell Biol. 128, 647-660.

Andersen, I.S., Voie, Ø.A., Fonnum, F., Mariussen, E., 2009. Effects of methyl mercury in combination with polychlorinated biphenyls and brominated flame retardants on the uptake of glutamate in rat brain synaptosomes: a mathematical approach for the study of mixtures. Toxicol. Sci. 112, 175-184.

Austin, M.E., Kasturi, B.S., Barber, M., Kannan, K., MohanKumar, P.S., MohanKumar, S.M., 2003. Neuroendocrine effects of perfluorooctane sulfonate in rats. Environ. Health. Perspect. 111, 1485-1489. Bliss, T.V., Collingridge, G.L., 1993. A synaptic model of memory: long-term potentiation in the hippocampus. Nature. 361, 31-39.

Borg, D., Bogdanska, J., Sundström, M., Nobelb, S., Håkanssona, H., Bergmanc, Å., DePierreb, J.W., Halldina, K., Bergströmd, U., 2011. Tissue distribution of 35S-labelled perfluorooctane sulfonate in adult mice after oral exposure to a low environmentally relevant dose or a high experimental dose. Toxicology. 284, 54-62.

Bukalo, O., Fentrop, N., Lee, A.Y., Salmen, B., Law, J.W.S., Wotjak, C.T., Schweizer1, M., Dityatev, A., Schachner, M., 2004. Conditional ablation of the neural cell adhesion molecule reduces precision of spatial learning, long-term potentiation, and depression in the CA1 subfield of mouse hippocampus. J Neurosci. 24, 1565-1577.

Butenhoff, J.L., Ehresman, D.J., Chang, S.C., Parker, G.A., Stump, D.G., 2009. Gestational and lactational exposure to potassium perfluorooctanesulfonate $\left(\mathrm{K}^{+} \mathrm{PFOS}\right)$ in rats: Developmental neurotoxicity. Reprod. Toxicol. 27, 319-330.

Cao, G.F., Liu, Y., Yang, W., Wan, J., Yao, J., Wan, Y., Jiang, Q., 2011. Rapamycin sensitive mTOR 
activation mediates nerve growth factor (NGF) induced cell migration and pro-survival effects against hydrogen peroxide in retinal pigment epithelial cells. Biochem. Biophys. Res. Commun. 414, 499-505. Chang, S.C., Ehresman, D.J., Bjork, J.A., Wallace, K.B., Parker, G.A., Stump, D.G., Butenhoff, J.L., 2009. Gestational and lactational exposure to potassium perfluorooctanesulfonate $\left(\mathrm{K}^{+} \mathrm{PFOS}\right)$ in rats: Toxicokinetics, thyroid hormone status, and related gene expression. Reprod. Toxicol. 27, 387-399.

Dityatev, A.; Schachner, M., 2003. Extracellular matrix molecules and synaptic plasticity. Nat. Rev. Neurosci. 4, 456-468.

Fields, R.D., Itoh, K., 1996. Neural cell adhesion molecules in activity-dependent development and synaptic plasticity. Trends. Neurosci. 19, 473-480.

Fischer, C., Fredriksson, A., Eriksson, P., 2008. Coexposure of neonatal mice to a flame retardant PBDE 99 (2, 2', 4, 4', 5-pentabromodiphenyl ether) and methyl mercury enhances developmental neurotoxic defects. Toxicol. Sci. 101, 275-285.

Fischer, W., Bjorklund, A., Chen, K., Gage, F.H., 1991. NGF improves spatial memory in aged rodents as a function of age. J. Neurosci. 11, 1889-1906.

Fuentes, S., Colomina, M.T., Vicens, P., Franco-Pons, N., Domingo, J.L., 2007a. Concurrent exposure to perfluorooctane sulfonate (PFOS) and restraint stress during pregnancy in mice: effects on postnatal development and behavior of the offspring. Toxicol. Sci. 98, 589-598.

Fuentes, S., Vicens, P., Colomina, M.T., Domingo, J.L., 2007b. Behavioral effects in adult mice exposed to perfluorooctane sulfonate (PFOS). Toxicology. 242, 123-129.

Giesy, J.P., Kannan, K., 2001. Global distribution of perfluorooctane sulfonate in wildlife. Environ. Sci. Technol. 35, 1339-1342.

Gygi, S.P., Rochon, Y., Franza, R.B., Aebersold, R., 1999. Correlation between protein and mRNA 
abundance in yeast. Mol. Cell. Biol. 19, 1720-1730.

Hansen, K.J., Clemen, L.A., Ellefson, M.E., Johnson, H.O., 2001. Compound-specific, quantitative characterization of organic fluorochemicals in biological matrices. Environ. Sci. Technol. 35, 766-770.

Heldt, S.A., Stanek, L., Chhatwal, J.P., Ressler, K.J., 2007. Hippocampus-specific deletion of BDNF in adult mice impairs spatial memory and extinction of aversive memories. Mol. Psychiatr. 12, 656-670.

Hughes, P.E., Alexi, T., Walton, M., Williams, C.E., Dragunow, M., Clark, R.G., Gluckman, P.D., 1999. Activity and injury-dependent expression of inducible transcription factors, growth factors and apoptosis-related genes within the central nervous system. Prog. Neurobiol. 57, 421-450.

Jin, Y.H., Liu, W., Sato, I., Nakayama, S.F., Sasaki, K., Saito, N., Tsuda, S., 2009. PFOS and PFOA in environmental and tap water in China. Chemosphere. 77, 605-611.

Johansson, N., Eriksson, P., Viberg, H., 2009. Neonatal exposure to PFOS and PFOA in mice results in changes in proteins which are important for neuronal growth and synaptogenesis in the developing brain. Toxicol. Sci. 108, 412-418.

Johansson, N., Fredriksson, A., Eriksson, P., 2008. Neonatal exposure to perfluorooctane sulfonate (PFOS) and perfluorooctanoic acid (PFOA) causes neurobehavioural defects in adult mice. Neurotoxicology. 29: 160-169.

Kemp, S.W., Webb, A.A., Dhaliwal, S., Syed, S., Walsh, S.K., Midha, R., 2011. Dose and duration of nerve growth factor (NGF) administration determine the extent of behavioral recovery following peripheral nerve injury in the rat. Exp. Neurol. 229, 460-470.

Korte, M., Carroll, P., Wolf, E., Brem, G., Thoenen, H., Bonhoeffer, T., 1995. Hippocampal long-term potentiation is impaired in mice lacking brain-derived neurotrophic factor. Proc. Natl. Acad. Sci. 92, 8856-8860. 
Lau, C., Anitole, K., Hodes, C., Lai, D., Pfahles, H.A., Seed, J., 2007. Perfluoroalkyl acids: a review of monitoring and toxicological findings. Toxicol. Sci. 99, 366-394.

Lau, C., Thibodeaux, J.R., Hanson, R.G., Rogers, J.M., Grey, B.E., Stanton, M.E., Butenhoff, J.L., Stevenson, L.A., 2003. Exposure to perfluorooctane sulfonate during pregnancy in rat and mouse. II. Postnatal evaluation. Toxicol. Sci. 74, 382-392.

Liu, X., Liu, W., Jin, Y., Yu, W., Liu, L., Yu, H., 2010a. Effects of subchronic perfluorooctane sulfonate exposure of rats on calcium-dependent signaling molecules in the brain tissue. Arch. Toxicol. 84, 471-479.

Liu, X., Liu, W., Jin, Y., Yu, W., Wang, F., Liu, L., 2010b. Effect of gestational and lactational exposure to perfluorooctanesulfonate on calcium-dependent signaling molecules gene expression in rats' hippocampus. Arch. Toxicol. 84, 71-79.

Livak, K.J., Schmittgen, T.D., 2001. Analysis of relative gene expression data using real-time quantitative PCR and the $2^{-\Delta C_{T}}$ method. Methods. 25, 402-408.

Luebker, D.J., Case, M.T., York, R.G., Moore, J.A., Hansen, K.J., Butenhoff, J.L., 2005. Two-generation reproduction and crossfoster studies of perfluorooctanesulfonate (PFOS) in rats. Toxicology. 215, 126-148.

Morris, R.G.M., Garrud, P., Rawlins, J.N.P., O'Keefe, J., 1982. Place navigation impaired in rats with hippocampal lesions. Nature. 297, 681-683.

Maier, T., Güell, M., Serrano, L., 2009. Correlation of mRNA and protein in complex biological samples. FEBS Lett. 583 (2009) 3966-3973.

Medina, C.O., Lattin, C.R., McVey, M., Romero, L.M., 2013. There is no correlation between glucocorticoid receptor mRNA expression and protein binding in the brains of house sparrows (Passer 
domesticus). Gen. Comp. Endocr. 193, 27-36.

Nie, L., Wu G., Zhang, W., 2006. Correlation between mRNA and protein abundance in Desulfovibrio vulgaris: a multiple regression to identify sources of variations. Biochem. Bioph. Res. Co. 339, 603-610

Nussbaum, R.L., Ellis, C.E., 2003. Alzheimer's disease and Parkinson's disease. N. Engl. J. Med. 348, 1356-1364.

Oestreicher, A.B., De Graan, P.N.E., Gispen, W.H., Verhaagen, J., Schrama, L.H., 1997. B-50, the growth associated protein-43: Modulation of cell morphology and communication in the nervous system. Prog. Neurobiol. 53, 627-686.

Patterson, S.L., Grover, L.M., Schwartzkroin, P.A., Bothwell, M., 1992. Neurotrophin expression in rat hippocampal slices: a stimulus paradigm inducing LTP in CA1 evokes increases in BDNF and NT-3 mRNAs. Neuron. 9, 1081-1088.

Petridis, A.K., El Maarouf, A., Rutishauser, U., 2004. Polysialic acid regulates cell contact-dependent neuronal differentiation of progenitor cells from the subventricular zone. Dev. Dyn. 230, 675-684.

Polesskaya, O.O., Fryxell, K.J., Merchant, A.D., Locklear, L.L., Ker, K., McDonald, C.G., Eppolito, A.K., Smith, L.N., Wheeler, T.L., Smith, R.F., 2007. Nicotine causes age-dependent changes in gene expression in the adolescent female rat brain. Neurotoxicol. Teratol. 29, 126-140.

Semple, B.D., Blomgren, K., Gimlin, K., Ferriero, D.M., Noble-Haeusslein, L.J., 2013. Brain development in rodents and humans: Identifying benchmarks of maturation and vulnerability to injury across species. Prog. Neurobiol. 106-107, 1-16.

Thompson, J., Eaglesham, G., Reungoat, J., Poussade, Y., Bartkow, M., Lawrence, M., Mueller, J.F., 2011. Removal of PFOS, PFOA and other perfluoroalkyl acids at water reclamation plants in South 
East Queensland Australia. Chemosphere. 82, 9-17.

Wang, F., Liu, W., Jin, Y., Dai, J., Yu, W., Liu, X., Liu, L., 2010. Transcriptional effects of prenatal and neonatal exposure to PFOS in developing rat brain. Environ. Sci. Technol. 44, 1847-1853.

Wang, F., Liu, W., Jin, Y., Dai, J., Zhao, H., Xie, Q., Liu, X., Yu, W., Ma, J., 2011. Interaction of PFOS and BDE-47 co-exposure on thyroid hormone levels and TH-related gene and protein expression in developing rat brains. Toxicol. Sci. 121, 279-291.

Wang, F., Liu, W., Ma, J., Yu, M., Jin, Y., Dai, J., 2012. Prenatal and neonatal exposure to perfluorooctane sulfonic acid results in changes in miRNA expression profiles and synapse associated proteins in developing rat brains. Environ. Sci. Technol. 46, 6822-6829.

Yu, W., Liu, W., Jin, Y., Liu, X., Wang, F., Liu, L., Nakayama, S.F., 2009. Prenatal and postnatal impact of perfluorooctane sulfonate (PFOS) on rat development: a cross-Foster study on chemical burden and thyroid hormone systemEnviron. Sci. Technol. 43, 8416-8422

Zhang, Z., Zhang, H., Du, B., Chen, Z., 2012. Neonatal handling and environmental enrichment increase the expression of GAP-43 in the hippocampus and promote cognitive abilities in prenatally stressed rat offspring. Neurosci. Lett. 522, 1-5. 
Graphical Abstracts (for review)
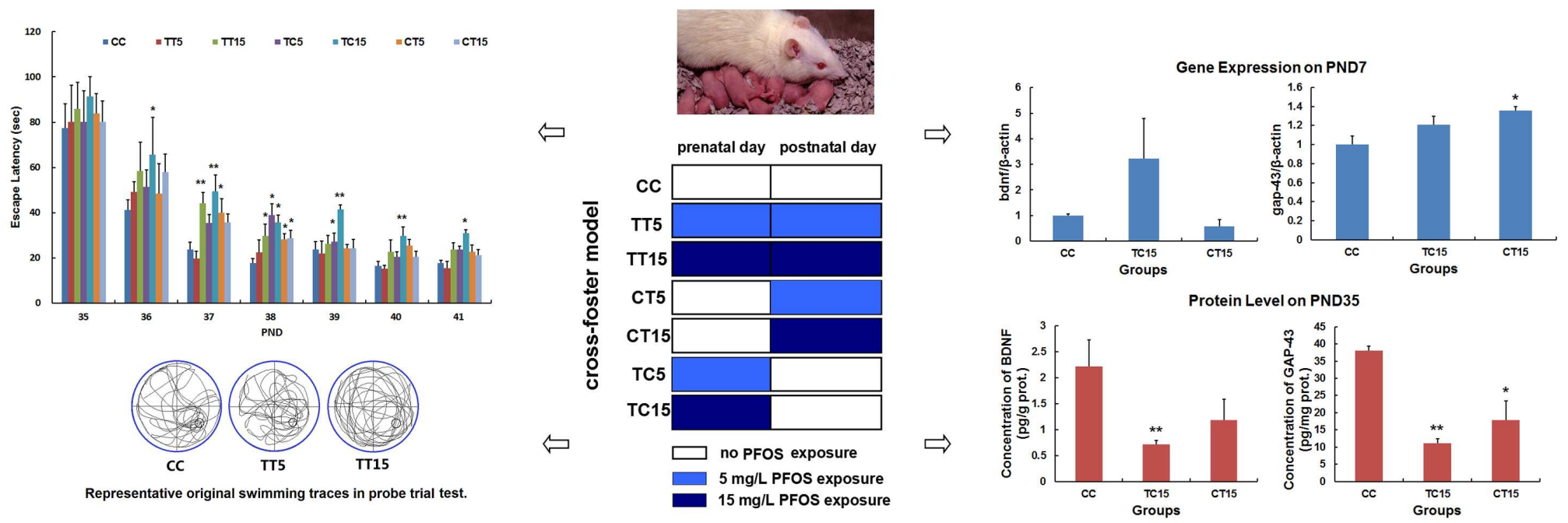